\title{
Потребление кислорода как показатель готовности к операции
}

\author{
3.А. Зарипова, А.С. Ткачук, А.В. Тишков, Л.В. Страх \\ Первый Санкт-Петербургский государственный медицинский университет \\ имени академика И.П. Павлова, \\ Россия, 197022, г. Санкт-Петербург, ул. Льва Толстого, 6-8 \\ E-mail: realzulya76@gmail.com
}

\begin{abstract}
Аннотация. В статье представлены результаты исследования потребления кислорода (ПК) на физической нагрузке у пациентов онкологического профиля. Нами было отмечено, что в условиях покоя ПК было практически одинаковым у всех обследуемых. В ходе кардиореспираторного нагрузочного тестирования пациенты 50-60 лет показали более значимый прирост ПК при работе в аэробных и анаэробных условиях по сравнению со старшей возрастной группой при идентичном времени нагрузки. При том что анаэробные условия, независимо от возраста, значимо снижали ПК и ограничивали работоспособность, само достижение анаэробного порога в старшей возрастной группе происходило на меньших мощностях нагрузки. Кроме того, ПК на уровне анаэробного порога и на пике нагрузки у них было значимо меньше. Поскольку снижение ПК в хирургии ассоциируется со значительным увеличением послеоперационной смертности от разных причин, проведение эргоспирометрии у пациентов необходимо для решения вопроса о готовности пациента к хирургическому вмешательству. Этот показатель может лечь в основу стратификации рисков развития неблагоприятных событий, что требует дополнительных исследований.
\end{abstract}

Ключевые слова: потребление кислорода, нагрузочное тестирование, предоперационная оценка, анаэробный порог.

Для цитирования: Зарипова 3.А., Ткачук А.С., Тишков А.В., Страх Л.В. 2020. Потребление кислорода как показатель готовности к операции. Актуальные проблемы медицины, 43 (3): 431-440. DOI: 10.18413/2687-0940-2020-43-3-431-440

\section{Oxygen consumption as an indicator of readiness for surgery}

\author{
Zulfiia A. Zaripova, Anastasiya S. Tkachuk, Artem V. Tishkov, Liubov V. Strakh \\ Pavlov First Saint Petersburg State Medical University, \\ 6-8 Lev Tolstoy St., Saint Petersburg, 197022, Russia \\ E-mail: realzulya76@gmail.com
}

\begin{abstract}
The article presents the results of a study of oxygen consumption (OC) during physical activity in patients with lung cancer. We noted that the OC was almost the same in all subjects at rest. During cardiorespiratory exercise testing, patients aged 50-60 years showed a more significant increase in OC when working in aerobic and anaerobic conditions, compared to the older age group, with identical load times. Despite the fact that anaerobic conditions, regardless of age, significantly reduced OC and limited performance, the achievement of the anaerobic threshold in the older age group occurred at lower load capacities. In addition, they had significantly less OC at the anaerobic threshold and peak load. Since a decrease in OC in surgery is associated with a significant increase in postoperative mortality from various causes, ergospirometry in patients is necessary to address the question of the patient's readiness for surgery. This indicator may form the basis for stratifying the risks of adverse events, which requires additional research.
\end{abstract}

Keywords: oxygen consumption, exercise testing, preoperative assessment, anaerobic threshold. 
For citation: Zaripova Z.A., Tkachuk A.S., Tishkov A.V., Strakh L.V. 2020. Oxygen consumption as an indicator of readiness for surgery. Challenges in Modern Medicine, 43 (3): 431-440 (in Russian). DOI: $10.18413 / 2687-0940-2020-43-3-431-440$

\section{Введение}

С учётом того, что увеличивается возраст пациентов, которым планируется оперативное вмешательство, растёт и число осложнений в послеоперационном периоде, поскольку пожилые имеют сниженные функциональные резервы для восстановления [Суркова и др., 2015; Wasserman, 1993; Jose, Maurizio, 2014; Older, Levett, 2017; Levett et al., 2018]. В состоянии покоя пациент может не предъявлять никаких жалоб, и проводимые инструментальные исследования не всегда выявляют проблему, что даёт основание допустить его до операции [Кербиков и др., 2012; Суркова и др., 2015; ATS/ACCP, 2003; Palange et al., 2007; Agnew, 2010; Wasserman et al., 2012]. Однако патология может быть скрытой, и она проявится только в условиях операционного стресса и нагрузки [Суркова и др., 2015; Jose, Maurizio, 2014; Levett et al., 2018], поскольку повышается метаболический запрос, который может быть удовлетворён только при состоятельности всех компенсаторно-приспособительных механизмов [Ватутин и др., 2016; Agnew, 2010]. В этой связи очевидна значимость кардиореспираторного нагрузочного тестирования (КАРЕН-Т) [Кербиков и др., 2012; Суркова и др., 2015; Ватутин и др., 2016; ATS/ACCP, 2003; Agnew, 2010; Jose, Maurizio, 2014; Guazzi et al., 2016; Older, Levett, 2017; Levett et al., 2018; Reeves et al., 2018]. КАРЕН-Т - это метод эргоспирометрии, который даёт объективную информацию о потреблении кислорода при нагрузке, на основании которого определяют адаптивные возможности организма, поскольку одновременно оценивается взаимодействие основных жизнеобеспечивающих систем [Кербиков и др., 2012; Суркова и др., 2015; Ватутин и др., 2016; ATS/ACCP, 2003; Agnew, 2010; Jose, Maurizio, 2014; Guazzi et al., 2016; Older, Levett, 2017; Levett et al., 2018; Reeves et al., 2018]. Максимальное потребление кислорода (МПК) в ряде исследований признается основным показателем готовности индивидуума к операции [Wasserman et al., 2012; Reeves et al., 2018], что нашло своё отражение в рекомендациях по ведению пациентов, которым планируется хирургическое вмешательство [Суркова и др., 2015; Ватутин и др., 2016; Weisman, Zeballos, 2001; ATS/ACCP, 2003; Smith, Stonell, 2009; Balady et al., 2010; Jose, Maurizio, 2014; Guazzi et al., 2016; Levett et al., 2018]. Однако не все могут достигать МПК в силу разных причин, в том числе из-за возраста [Тавровская, 2007; Суркова и др., 2015; Agnew, 2010]. В этом случае оценивается прирост потребления кислорода и его значение на анаэробном пороге и пике нагрузки, что также показывает резервные возможности организма [Мустафина и др., 2013; Carlson, 1995; Palange et al., 2007]. Изучение потребления кислорода в ходе КАРЕН-Т в возрастном аспекте является перспективным направлением для оценки рисков послеоперационных осложнений в торакальной хирургии [Older, Levett, 2017; Levett et al., 2018], что и послужило основанием для проведения данного исследования.

Цель исследования - сравнить уровень потребления кислорода у пациентов среднего и пожилого возраста в предоперационном периоде.

\section{Материалы и методы исследования}

В статистическую обработку включены данные пациентов без подтверждённой сопутствующей патологии, которые поступили в клинику ПСПбГМУ им. И.П. Павлова $(\mathrm{n}=91)$ для хирургического лечения новообразования лёгкого. Среди диагнозов: аденокарцинома легкого, периферический и центральный плоскоклеточный рак лёгкого/бронхов. Степень поражения pT2b-3,N1-2,M0, отсутствие отдалённых метастазов 
по данным обследования. К средней возрастной группе $(\mathrm{n}=42)$ относили мужчин и женщин от 50 до 60 лет, к группе пожилого возраста $(\mathrm{n}=49)$ - от 61 до 70 лет (табл. 1).

Критерии включения: информированное согласие на участие в исследовании, подтверждённый диагноз новообразования лёгкого, планируемое оперативное вмешательство, отсутствие противопоказаний к выполнению КАРЕНТ-Т.

Критерии невключения: постоянный приём бета-блокаторов (из-за невозможности достижения максимальной расчётной частоты сердечных сокращений), выявление признаков обструкции при спирометрии в покое, значимая анемия (гемоглобин ниже 110 г/л), ожирение (индекс массы тела более 30).

Всем пациентам проводилось КАРЕН-Т на аппарате Cortex с применением модуля Custo Diagnostic (CustoMed $\mathrm{GmbH}$ ), интегрированного с газоанализатором MetaLyzer ${ }^{\circledR}$ 3B/MetaLizer ${ }^{\circledR}$ II и оценкой в программе MetaSoft-Studio (MetaSoftSoftware, CORTEX CPET system) с использованием вертикального велоэргометра Ergoline по единому стандартному протоколу рамповой нагрузки «Предоперационная оценка» с постоянным приростом в 10 Ватт за каждую минуту педалирования [Тавровская, 2007; ATS/ACCP, 2003; Balady et al., 2010; Levett et al., 2018]. Все параметры автоматически в режиме «breath-by-breath» записывались в базу данных, доступную для выгрузки и анализа.

Все условия при проведении кардиореспираторного нагрузочного тестирования были соблюдены согласно практическим рекомендациям по велоэргометрии [Тавровская, 2007; ATS/ACCP, 2003]. В режиме реального времени оценивали показатели гемодинамики и газоанализа в периоды покоя (3 мин), свободного педалирования (3 мин), нагрузки (8-15 мин) до достижения 75-85\% от максимальной расчётной частоты сердечных сокращений и восстановления (3 мин). Внутри нагрузочного этапа выделили 3 периода: малые (от 10 до 40 Вт), средние (от 41 до 70 Вт) и высокие (от 71 до 90 Вт) нагрузки с измерениями на конец периода. Также выделяли период работы в аэробных (до достижения анаэробного порога) и в анаэробных условиях. Определение анаэробного порога (АП) проводилось визуальным способом по кривым и наклонам. Критерием правильности выбранного участка служил дыхательный коэффициент (ДК), равный 1,0 [Тавровская, 2007; Кербиков и др., 2012; Мустафина и др., 2013; Ватутин и др., 2016; Balady et al., 2010].

Основной оцениваемый параметр в динамике: потребление кислорода (ПК, $\mathrm{V}^{\prime} \mathrm{O}_{2}$, л/мин) и его производное - относительное потребление кислорода, с учётом массы тела ( $\mathrm{V}^{\prime} \mathrm{O}_{2} /$ кг, мл/мин/кг) как более точный параметр [Ватутин и др., 2016]. На высоте нагрузки, которую пациенты в состоянии были выполнить, мы оценивали «пиковое потребление кислорода» (V'O $\mathrm{V}_{2}$ peak) [Carlson, 1995]. Кроме того, оценивали достижение расчётных значений мощности.

Во время проведения теста производили мониторинг классических переменных [Ватутин и др., 2016]: клиническую реакцию испытуемого на физическую нагрузку, гемодинамический ответ и изменения ЭКГ.

Все пациенты были сопоставимы по исходным параметрам (табл. 1).

Внутри каждой возрастной группы мужчины и женщины имели значимые отличия по росту, массе тела и ППТ ( $<<0,001)$, как видно из табл. 1 , в связи с чем сравнение между ними в дальнейшем не производилось.

Сравнение по параметрам выполнялось между разными возрастными группами по гендерному признаку. Различия по росту, массе тела, ИМТ и ППТ (средний и пожилой возраст) были недостоверны в обеих гендерных когортах, p>0,05. Предтестовые измерения по гемодинамике и функции дыхания в них также не имели между собой статистически значимых отличий, $\mathrm{p}>0,05$, за исключением частоты дыхания у женщин $(\mathrm{p}=0,02)$.

Статистика. Статистическое описание мерных данных для нормального распределения приведено в виде $\mathrm{M}(\mathrm{SD})$ или $\mathrm{Me}[\mathrm{x} 1$; х3] при ненормальном распределении. 
Проверка нормальности и согласованности проводилась с помощью критерия ШапироУилка. Различия между двумя выборками с измеряемыми атрибутами определялись при помощи критерия Стьюдента или рангового критерия Манна - Уитни. Для сравнения зависимых выборок использовали парный параметрический критерий Стьюдента и парный ранговый критерий Вилкоксона. При проверке гипотез критический уровень значимости принимали равным 0,05 .

Таблица 1

Table 1

Сравнительная характеристика (состояние покоя, предтестовые измерения) пациентов, включённых в исследование

Comparative characteristics (resting state, pre-test measurements) of patients included in the study

\begin{tabular}{|l|c|c|c|c|}
\hline \multirow{2}{*}{ Параметры } & \multicolumn{2}{|c|}{$\begin{array}{c}\text { Средний возраст } \\
(50-60 \text { лет) }\end{array}$} & \multicolumn{2}{c|}{$\begin{array}{c}\text { Пожилой возраст } \\
(61-70 \text { лет) }=49\end{array}$} \\
\cline { 2 - 5 } & $\begin{array}{c}\text { Группа 1, } \\
\text { мужчины, } \\
(\mathrm{n}=27)\end{array}$ & $\begin{array}{c}\text { Группа 2, } \\
\text { женщины, } \\
(\mathrm{n}=15)\end{array}$ & $\begin{array}{c}\text { Группа 3, } \\
\text { мужчины, } \\
(\mathrm{n}=29)\end{array}$ & $\begin{array}{c}\text { Группа 4, } \\
\text { женщины, } \\
(\mathrm{n}=20)\end{array}$ \\
\hline Возраст, лет & $55,67(3,10)$ & $55,67(2,65)$ & $65,66(2,35)$ & $66,65(2,31)$ \\
\hline Масса тела, кг & $74,41(9,64)$ & $65,10(6,19)$ & $73,38(9,86)$ & $62,50(9,32)$ \\
\hline Рост, см & $173,44(7,32)$ & $164,00(4,83)$ & $172,76(6,32)$ & $160,95(4,96)$ \\
\hline ППТ, м ${ }^{2}$ & $53,02(4,17)$ & $48,11(2,35)$ & $53,65[49,07 ; 55,97]$ & $46,59(3,80)$ \\
\hline ИМТ & $24,70(2,54)$ & $24,20(2,43)$ & $24,55(2,31)$ & $24,20(3,04)$ \\
\hline ЧСС, в мин & $85,49(15,32)$ & $86,02(14,61)$ & $84,39(12,27)$ & $83,07(13,70)$ \\
\hline САД, мм рт. ст. & $136,21(12,75)$ & $125,50(11,50)$ & $134,80(14,13)$ & $131,44(16,91)$ \\
\hline ДАД, мм рт. ст. & $84,57(8,99)$ & $87,50(10,50)$ & $84,90(7,49)$ & $79,00(8,91)$ \\
\hline ЧД, в мин & $19,50(5,88)$ & $19,14[16,19 ;$ & $20,01(5,65)$ & $19,79(4,18)$ \\
\hline ДО, л & $0,85[0,65 ; 0,93]$ & $0,59[0,40 ; 0,71]$ & $0,87[0,67 ; 0,94]$ & $0,58[0,50 ; 0,76]$ \\
\hline ЖЕЛ, л & $3,56(0,65)$ & $2,88(0,52)$ & $3,22(0,66)$ & $2,59(0,54)$ \\
\hline ОФВ1, л & $2,72(0,72)$ & $2,38(0,45)$ & $2,58(0,57)$ & $2,05(0,39)$ \\
\hline SрО $2, \%$ & $96,69[94,41 ; 97,94]$ & $98,01[95,52 ; 98]$ & $97,41[95 ; 98]$ & $96,09[94,35 ; 97,5]$ \\
\hline
\end{tabular}

Примечание: данные приведены в формате среднее (стандартное отклонение) для нормально распределённой выборки и в формате медиана [нижний квартиль; верхний квартиль] для ненормального распределения; сравнение осуществлялось с помощью критериев Стьюдента и Манна - Уитни.

Сокращения: ППТ - площадь поверхности тела, ИМТ - индекс массы тела, ЧСС - частота сердечных сокращений, САД - систолическое артериальное давление, ДАД - диастолическое артериальное давление, ЧД - частота дыхания, ДО - дыхательный объем, ЖЕЛ - жизненная ёмкость лёгких, ОФВ1 - объем форсированного выдоха за первую секунду, $\mathrm{SpO}_{2}$ - сатурация (насыщение гемоглобина кислородом)

\section{Результаты и их обсуждение}

Глобальное (общее) потребление кислорода (ПК, V'О 2 , л/мин) как отражение адаптивных возможностей организма в целом [Carlson, 1995; ATS/ACCP, 2003; Palange et al., 2007] в состоянии покоя во всех группах было практически одинаковым (табл. 2), что ещё раз доказывает необходимость нагрузочных проб, чтобы увидеть истинную картину функциональных резервов организма. В конце периода малых нагрузок (на 40 Вт) также не было выявлено значимых отличий (табл. 2), однако мы наблюдали неравномерный прирост показателя на 40 Вт по отношению к состоянию покоя: так, у мужчин он составил $+60,45 \%$ и $+68,60 \%$ по 1 гр. и 3 гр. соответственно. В это же время у женщин прирост был значимо 
больше: $+162,21 \%$ во 2 гр. и $+95,46 \%$ в 4 гр., как показано в табл. 2, что показывает наличие межгендерной разницы в механизмах адаптации к нагрузке [Hossock, Bruce, 1982].

Таблица 2

Table 2

Изменение глобального потребления кислорода на различных этапах нагрузочного тестирования Changes in global oxygen consumption at various stages of load testing

\begin{tabular}{|c|c|c|c|c|c|c|}
\hline \multirow{2}{*}{$\begin{array}{c}\text { Глобальное } \\
\text { потребление } \\
\text { кислорода, } \mathrm{V}^{\prime} \mathrm{O}_{2} \text {, } \\
\text { л/мин }\end{array}$} & \multicolumn{2}{|c|}{ Мужчины } & & \multicolumn{2}{|c|}{ Женщины } & \\
\hline & $\begin{array}{c}\text { Группа } 1 \\
(\mathrm{n}=27)\end{array}$ & $\begin{array}{c}\text { Группа } 3 \\
(\mathrm{n}=29)\end{array}$ & & $\begin{array}{c}\text { Группа } 2 \\
(\mathrm{n}=15)\end{array}$ & $\begin{array}{c}\text { Группа } 4 \\
(\mathrm{n}=20)\end{array}$ & \\
\hline Покой & $0,39(0,12)$ & $0,40(0,12)$ & $p=0,79$ & $0,23[0,20 ; 0,27]$ & $0,31[0,22 ; 0,46]$ & $p=0,07$ \\
\hline $\begin{array}{l}\text { Свободное } \\
\text { педалирование }\end{array}$ & $0,45(0,07)$ & $0,46(0,07)$ & $p=0,73$ & $0,44(0,09)$ & $0,42(0,07)$ & $p=0,50$ \\
\hline $\begin{array}{l}\text { Конец периода малых } \\
\text { нагрузок, } 40 \text { Вт } \\
\text { (прирост по отноше- } \\
\text { нию к покою) }\end{array}$ & $\begin{array}{l}0,62(0,11) \\
(+60,45 \%)\end{array}$ & $\begin{array}{l}0,67(9,10) \\
(+68,60 \%)\end{array}$ & $p=0,11$ & $\begin{array}{c}0,71(0,09) \\
(+162,21 \%)\end{array}$ & $\begin{array}{l}0,67(0,11) \\
(+95,46 \%)\end{array}$ & $p=0,22$ \\
\hline $\begin{array}{l}\text { Конец периода сред- } \\
\text { них нагрузок, } 70 \text { Вт }\end{array}$ & $0,96(0,17)$ & $0,93(0,12)^{*}$ & $\mathrm{p}=0,44$ & $0,91(0,08)^{*}$ & - & \\
\hline $\begin{array}{l}\text { Конец периода высо- } \\
\text { ких нагрузок, } 90 \text { Вт }\end{array}$ & $1,18(0,38)^{*}$ & $1,20(0,31)^{*}$ & $\mathrm{p}=0,82$ & $1,16(0,07)^{*}$ & - & \\
\hline Анаэробный порог & $1,01[0,88 ; 1,21]$ & $0,91(0,18)$ & $p=0,03$ & $0,91(0,19)$ & $0,79(0,12)$ & $p=0,04$ \\
\hline Пик нагрузки & $1,14[1,01 ; 1,38]$ & $1,03(0,12)$ & $p=0,01$ & $1,04(0,17)$ & $0,89(0,16)$ & $p=0,01$ \\
\hline $\begin{array}{l}\text { Восстановление } \\
1 \text { минута }\end{array}$ & $1,02(0,21)$ & $0,91(0,19)$ & $p=0,05$ & $0,89(0,13)$ & $0,76(0,14)$ & $p=0,008$ \\
\hline $\begin{array}{l}\text { Восстановление } \\
2 \text { минута }\end{array}$ & $0,65(0,13)$ & $0,56(0,11)$ & $p=0,01$ & $0,55(0,09)$ & $0,47(0,09)$ & $p=0,03$ \\
\hline $\begin{array}{l}\text { Восстановление } \\
3 \text { минута }\end{array}$ & $0,46(0,11)$ & $0,41[0,35 ; 0,43]$ & $p=0,14$ & $0,39(0,08)$ & $0,34(0,05)$ & $p=0,02$ \\
\hline
\end{tabular}

Примечание: данные приведены в формате среднее (стандартное отклонение) для нормально распределённой выборки, и в формате медиана [нижний квартиль; верхний квартиль] для ненормального распределения; сравнение осуществлялось с помощью критериев Стьюдента и Манна - Уитни.

* Параметры после достижения анаэробного порога (анаэробные условия).

К концу периода средних нагрузок (на 70 Вт) только пациенты 1 гр. работали ещё в аэробных условиях, все остальные - в анаэробных. С учётом полученных результатов мы разделили всю нагрузку на аэробную (от 40 Вт до достижения АП) и анаэробную, которую пациенты выполняли от АП до максимально выполнимой, и далее сравнивали эти этапы. Рис. 1Б показывает, что прирост ПК в аэробных условиях во всех группах был значимо больше, чем в анаэробных ( $\mathrm{p}<0,01)$.

Основные различия между группами были выявлены на анаэробном пороге как у мужчин, так и у женщин ( $<<0,05$, табл. 2). При оценке относительного потребления кислорода ( $\mathrm{V}^{\prime} \mathrm{O}_{2} / \kappa г$, мл/мин/кг) [Ватутин и др., 2016], мы получили сопоставимые результаты, на АП также увидели отличия в группах: в 1 гр. 14,41 $(2,90)$ мл/мин/кг, в 3 гр. $12,48(2,46)$ мл/мин/кг $(\mathrm{p}=0,01)$; во 2 гр. 14,07 (2,41) мл/мин/кг и 13,00 [11,5; 13,5] мл/мин/кг в 4 гр., $\mathrm{p}=0,03$. Как видно из этих данных, все обследуемые пациенты на АП достигли значения $\mathrm{V}^{\prime} \mathrm{O}_{2} /$ кг в 11 мл/мин/кг, что, согласно ряду исследований, показывает низкие риски развития осложнений в послеоперационном периоде и позволяет выполнить любое оперативное вмешательство независимо от возраста [Суркова и др., 2015; Wasserman et al., 2012; Jose, Maurizio, 2014; Older, Levett, 2017; Reeves et al., 2018]. 

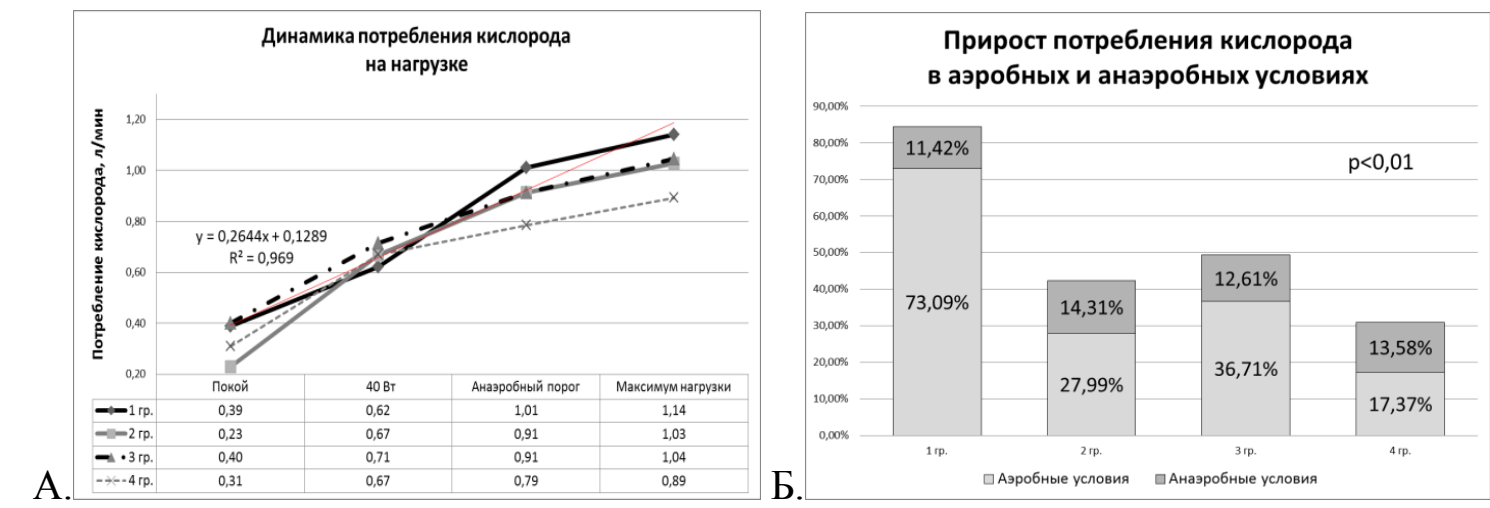

Рис. 1. А. Динамика потребления кислорода в группах. Для каждой группы анаэробный порог (АП) достигался на разной нагрузке, также как и максимальные значения нагрузки различались между собой (объяснения в тексте). При этом отмечался общий тренд прироста потребления кислорода от малых нагрузок до достижения АП, со снижением прироста после него (Б)

Fig. 1. A. Dynamics of oxygen consumption in groups. For each group, the anaerobic threshold (AT) was reached at different loads, as well as the maximum load values were different (explanations in the text). At the same time, there was a general trend of increase in oxygen consumption from low loads to AT, with a decrease in growth after it (B)

Однако следует отметить, что старшая возрастная группа достигла анаэробного порога (АП) на нагрузке 66,55 $(18,03)$ Вт (мужчины) и 46,87 $(8,06)$ Вт (женщины), что было статистически значимо раньше, по сравнению с более молодыми пациентами: $82,33(24,63)$ Вт 1 гр., $\mathrm{p}=0,009$, и 65,57 (13,01) Вт во 2 гр., $\mathrm{p}=0,0001$ (рис. 2). Нагрузка на конец исследования также отличалась внутри групп и составила для мужчин 1 гр. и 3 гр. $96,93(21,42)$ Вт и 79,34 (12,16) Вт соответственно $(\mathrm{p}=0,0004)$, для женщин 2 гр. $73,27(12,52)$ Вт, 4 гр. - 59,00 (12,87) Вт (p=0,003) (рис. 2). При этом расчётных значений мощности [Тавровская, 2007] пациенты не достигали (рис. 2).

C учётом недостижения пациентами максимального (для них возможного) потребления кислорода, мы учитывали не максимальное, а пиковое потребление кислорода (на пике нагрузки, $\mathrm{V}^{\prime} \mathrm{O}_{2}$ peak) [Carlson, 1995; Palange et al., 2007], которое для мужчин 1 гр. и 3 гр. было равно $1,14[1,01 ; 1,38]$ л/мин и $1,03(0,12)$ л/мин соответственно, $p=0,01$; для женщин 2 гр. 1,04 $(0,17)$ л/мин и 0,89 $(0,16)$ л/мин для 4 гр., $\mathrm{p}=0,01$, (табл. 2, рис. 1). При этом между $\mathrm{V}^{\prime} \mathrm{O}_{2}$ peak и мощностью нагрузки выявлена сильная корреляция: $\mathrm{rs}=0,872$ $(\mathrm{p}<0,001)$ для 1 гр., $\mathrm{r}=0,811(\mathrm{p}<0,001)$ для 3 гр., $\mathrm{r}=0,804(\mathrm{p}<0,001)$ для 2 гр., и $\mathrm{r}=0,808$ $(\mathrm{p}<0,001)$ для 4 гр. Кроме того, относительное потребление кислорода $\left(\mathrm{V}^{\prime} \mathrm{O}_{2} / \kappa г\right)$ на высоте нагрузки сильно и средне коррелировало с работоспособностью (WR/кг, Вт/кг), суммарным показателем физиологических возможностей организма, отражающим толерантность к физической нагрузке [ATS/ACCP, 2003; Wasserman et al., 2012]. Так, у пациентов 1 и 2 групп коэффициент корреляции составил $\mathrm{r}=0,906$ ( $\mathrm{p}<0,001)$ у мужчин и $\mathrm{r}=0,792(\mathrm{p}<0,001)$ у женщин, а в группах пожилых - rs $=0,926$ ( $\mathrm{p}<0,001)$ у мужчин и $\mathrm{rs}=0,771(\mathrm{p}<0,001)$ у женщин. Прирост работоспособности в аэробных условиях во всех группах был значимо больше, чем в анаэробных (рис. 2Б). Все изменения между этапами внутри групп были значимыми ( $<<0,001)$.

Время нагрузки у всех было сопоставимо и для 1 гр. составило 7:21 (1:41) мин, для 3 гр. - 7:01 (1:20) мин, p=0,42, для 2 гр. - 9:37 (1:43) мин, для 4 гр. $-8: 47$ (2:10) мин, $p=0,23$. Отметим, что пациенты 1 гр. и 2 гр. выполнили исходно бо́льшую нагрузку относительно сравниваемых с ними групп (рис. 2), то есть за одно и то же время нагрузочного этапа показали более высокую работоспособность. Окончание теста было связано с достижением 75-85\% от расчётной ЧСС и утомлением пациента. Признаков ишемии и иных причин, которые могли бы ограничить педалирование, зафиксировано не было. 


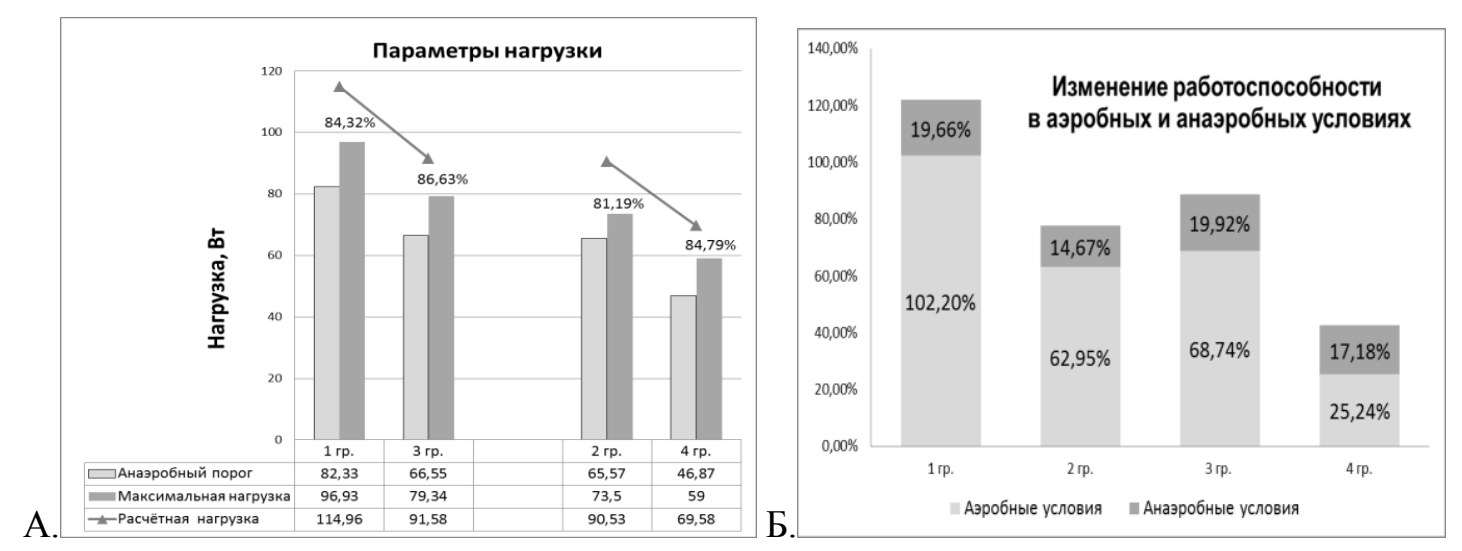

Рис. 2. А. Данные по нагрузке. Пациенты не достигали расчётных значений мощности нагрузки.

В старшей возрастной группе выполняемая нагрузка была меньше. Б. Динамика работоспособности в зависимости от работы в аэробных и анаэробных условиях

Fig. 2. A. Load data. Patients did not reach the calculated load capacity values. In the older age group, the load performed was less. B. dynamics of performance depending on work in aerobic and anaerobic conditions

Мы оценили потребление кислорода у пациентов онкологического профиля среднего и пожилого возраста в предоперационном периоде. В состоянии покоя (табл. 1) отличия не были выявлены, кроме того, сравниваемые группы имели исходно сопоставимые параметры гемодинамики и функции дыхания [Суркова и др., 2015; Palange et al., 2007]. Эргоспирометрия в условиях нагрузки на велоэргометре была необходима для оценки взаимодействия систем доставки и потребления кислорода [Weisman, Zeballos, 2001; ATS/ACCP, 2003; Palange et al., 2007; Wasserman et al., 2012; Guazzi et al., 2016]. Способность увеличивать потребление кислорода на нагрузке и достигать расчётных значений мощности показывает общие адаптационные возможности организма [Мустафина и др., 2013; Ватутин и др., 2016; Weisman, Zeballos, 2001; ATS/ACCP, 2003; Agnew, 2010; Balady et al., 2010; Jose, Maurizio, 2014; Guazzi et al., 2016; Levett et al., 2018] и зависит от возраста [Wasserman K., 1993]. В нашем исследовании пациенты в возрасте 50-60 лет показали более значимый прирост потребления кислорода и работоспособность при работе в аэробных и анаэробных условиях, по сравнению со старшей возрастной группой, при одинаковом времени нагрузки. Это важно учитывать, поскольку сниженное потребление кислорода в хирургии ассоциируется со значительным увеличением послеоперационной смертности от разных причин [Ватутин и др., 2016; Суркова и др., 2015].

Таким образом, возраст является значимым фактором, который ограничивает прирост потребления кислорода, определяющего возможность противостоять операционному стрессу. Важно учитывать этот показатель в плане обследования пациентов старше 60 лет, оценивая не только их данные в покое, но и резервные возможности при нагрузке. Потребление кислорода в возрастном аспекте в условиях аэробного и анаэробного метаболизма может иметь высокую практическую значимость и лечь в основу стратификации рисков перед операцией [Jose, Maurizio, 2014; Guazzi et al., 2016; Older, Levett, 2017; Levett et al., 2018] наряду с общепринятыми, что требует дополнительных исследований.

\section{Выводы}

1. Условия нагрузки показали значимую межвозрастную разницу в потреблении кислорода, которая не была выявлена в покое. У пациентов старше 60 лет уровень потребления кислорода снижается. Работоспособность сильно коррелирует со способностью увеличивать потребление кислорода на нагрузке и зависит от возраста. Снижение потребления кислорода приводит к ограничению работоспособности и возможности достижения расчётных мощностей. 
2. Работоспособность в анаэробных условиях значимо снижена и делает нецелесообразным продолжение нагрузки для пациента. Потребление кислорода на уровне анаэробного порога и на пике нагрузки у пациентов старшей возрастной группы значимо меньше по сравнению с более молодыми, что может указывать на их сниженные резервные возможности, которые потенциально могут влиять на течение периоперационного периода.

3. Возраст пациента старше 60 лет можно рассматривать как показание к оценке потребления кислорода на нагрузке перед операцией, а полученную информацию нужно учитывать при стратификации рисков развития послеоперационных осложнений и готовность пациента перенести хирургическое вмешательство. Эргоспирометрию следует назначать как дополнительный необходимый вариант обследования пациентов для оценки резервов организма.

\section{Список литературы}

1. Ватутин Н.Т., Смирнова А.С., Гасендич Е.С., Тов И.В. 2017. Современный взгляд на кардиопульмональное нагрузочное тестирование (обзор рекомендаций EACPR/AHA, 2016). Архив внутренней медицины. 1: 5-14. DOI: 10.20514/2226-6704-2017-7-1-5-14.

2. Кербиков О.Б., Аверьянов А.В., Борская Е.Н., Крутова Т.В. 2012. Кардиопульмональное нагрузочное тестирование в клинической практике. Журнал клиническая практика. 2: 58-70.

3. Мустафина М.Х., Черняк А.В. 2013. Кардиореспираторный нагрузочный тест // Атмосфера. Пульмонология и аллергология. 3: 56-62.

4. Рекомендации ESC/ESA по предоперационному обследованию и ведению пациентов при выполнении внесердечных хирургических вмешательств, 2014: пер. с англ. Суркова Е.А., Щукин Ю.В., Дупляков Д.В. Российский кардиологический журнал. 2015.8 (124): 7-66.

5. Тавровская Т.В. 2007. Велоэргометрия: практическое руководство для врачей. - СанктПетербург. - Издательство: Нео. 134 с.

6. American Thoracic Society, American College of Chest Physicians, ATS/ACCP Statement on Cardiopulmonary Exercise Testing. Am. J. Respir. Crit. Care. Med. 2003. 167: 211-277.

7. Agnew N. 2010. Preoperative cardiopulmonary exercise testing. N. Agnew. Continuing Education in Anaesth, Crit. Care \& Pain. 10 (2): 33-37.

8. Balady G.J., Arena R., Sietsema K. 2010. Clinician's Guide to Cardiopulmonary Exercise Testing in Adults: A Scientific Statement From the American Heart Association. Circulation. 122: 191-225. PMID: 20585013, DOI:10.1161/CIR.0b013e3181e52e69.

9. Carlson D.J. 1995. VO ${ }_{2} \max$ : The Gold Standard? Chest. 108 (3): 602-603. PMID:7656603, DOI:10.1378/chest.108.3.602.

10. Guazzi M., Arena R., Halle M. 2016. Focused Update: Clinical Recommendations for Cardiopulmonary Exercise Testing Data Assessment in Specific Patient Populations. Circulation. 133: 694-711.

11. Hossock K.F., Bruce R.A. 1982. Maximal cardiac function in sedentary normal men and women: comparison of age related changes. Appl. Physiol. 53: 799-804.

12. Jose L., Maurizio S. 2014. ESC/ESA Guidelines on non-cardiac surgery: cardiovascular assessment and management. The Joint Task Force on non-cardiac surgery: cardiovascular assessment and management of the European Society of Cardiology (ESC) and the European Society of Anaesthesiology (ESA). Eur. Heart. J. 35: 2383-2431.

13. Levett D.Z.H., Jack S., Swart M., Carlisle J., Wilson J., Snowden C., Riley M., Danjoux G., Ward S.A., Older P., Grocott M.P.W. 2018. Perioperative Exercise Testing and Training Society (POETTS) Perioperative Cardiopulmonary Exercise Testing (CPET): Consensus Clinical Guidelines on Indications, Organization, Conduct, and Physiological Interpretation. Br. J. Anaesth. 120 (3): 484-500. PMID: 29452805, DOI: 10.1016/j.bja.2017.10.020.

14. Older P.O., Levett D.Z.H. 2017. Cardiopulmonary Exercise Testing and Surgery. Ann. Am. Thorac. Soc. 1: S74-S83. DOI: 10.1513/AnnalsATS.201610-780FR.

15. Palange P., Ward S.A. 2007. Recommendations on the use of exercise testing in clinical practice. Eur. Respir. J. 29 (1): 185-209. DOI: 10.1183/09031936.00046906. 
16. Reeves T., Bates S., Sharp T., Richardson K., Bali S., Plumb J., Anderson H., Prentis J., Swart M., Levett D.Z.H. 2018. Perioperative Exercise Testing and Training Society (POETTS). Cardiopulmonary Exercise Testing (CPET) in the United Kingdom-a National Survey of the Structure, Conduct, Interpretation and Funding. Perioper. Med. (Lond). 7: 2-8. PMID: 29423173, DOI: 10.1186/s13741-0170082-3.

17. Smith T.B., Stonell C. 2009. Cardiopulmonary exercise testing as a risk assessment method in non-cardio-pulmonary surgery: a systematic review. Anaesthesia. 64 (8): 883-893.

18. Wasserman K. 1993. Preoperative evaluation of cardiovascular reserve in the elderly. Chest. 104 (3): 663-664. PMID: 8365269, DOI: 10.1378/chest.104.3.663.

19. Wasserman K., Hansen J.E., Sue D.Y., Stringer W.W., Sietsema K.E., Sun X-G., Whipp B.J. 2012. Principles of Exercise Testing and Interpretation: 5th edition. Lippincott Williams \& Wilkins. 899 p. ISBN-13: 978-1-60913-899-8.

20. Weisman I.M., Zeballos R.J. 2001. Clinical Exercise Testing. Clin. Chest. Med. 22 (4): 679-701. PMID: 11787659, DOI: 10.1016/s0272-5231(05)70060-5.

\section{References}

1. Vatutin N.T., Smirnova A.S., Gasendich E.S., Tov I.V. 2017. Sovremenniy vzglad na cardiopul'monal'noe nagruzochnoe testirovanie (obzor recomendaciy EACPR/AHA, 2016) [Modern view on cardiopulmonary load testing (review of EACPR/AHA recommendations, 2016)]. Arkhiv vnutrenney medicine, 1: 5-14. DOI: 10.20514/2226-6704-2017-7-1-5-14.

2. Kerbikov O.B., Averyanov A.V., Borskaya E.N., Krutova T.V. 2012. Cardiopul'monal'noe nagruzochnoe testirovanie $\mathrm{v}$ klinicheskoy praktike [Cardiopulmonary load testing in clinical practice]. Zhurnal klinicheskaya praktika, 2: 58-70.

3. Mustafina M.Kh., Chernyak A.V. 2013. Kardiorespiratorniy nagruzochniy test [Cardiorespiratory load test]. Atmosphere. Pulmonology and Allergology, 3: 56-62.

4. Recomendacii ESC/ESA po predoperacionnomu obsledovaniyu i vedeniyu pacientov pri vipolnenii vneserdechnikh khirurgicheskikh vmeshatel'stv, 2014. 2015 [Recommendations of the ESC/ESA on preoperative examination and management of patients during extra-cardiac surgical interventions, 2014: translation from English Surkova E.A., Shchukin Yu.V., Duplyakov D.V.]. Rossiyskiy kardiologicheskiy zhurnal, 8 (124): 7-66.

5. Tavrovskaya T.V. Veloergometriya: prakticheskoe rucovodstvo dlya vrachey [Veloergometry: a practical guide for doctors]. Saint Petersburg, Neo, $134 \mathrm{p}$.

6. American Thoracic Society, American College of Chest Physicians, ATS/ACCP Statement on Cardiopulmonary Exercise Testing. Am. J. Respir. Crit. Care. Med. 2003. 167: 211-277.

7. Agnew N. 2010. Preoperative cardiopulmonary exercise testing. N. Agnew. Continuing Education in Anaesth, Crit. Care \& Pain. 10 (2): 33-37.

8. Balady G.J., Arena R., Sietsema K. 2010. Clinician's Guide to Cardiopulmonary Exercise Testing in Adults: A Scientific Statement From the American Heart Association. Circulation. 122: 191-225. PMID: 20585013, DOI:10.1161/CIR.0b013e3181e52e69.

9. Carlson D.J. 1995. $\mathrm{VO}_{2} \max$ : The Gold Standard? Chest. 108 (3): 602-603. PMID:7656603, DOI:10.1378/chest.108.3.602.

10. Guazzi M., Arena R., Halle M. 2016. Focused Update: Clinical Recommendations for Cardiopulmonary Exercise Testing Data Assessment in Specific Patient Populations. Circulation. 133: 694-711.

11. Hossock K.F., Bruce R.A. 1982. Maximal cardiac function in sedentary normal men and women: comparison of age related changes. Appl. Physiol. 53: 799-804.

12. Jose L., Maurizio S. 2014. ESC/ESA Guidelines on non-cardiac surgery: cardiovascular assessment and management. The Joint Task Force on non-cardiac surgery: cardiovascular assessment and management of the European Society of Cardiology (ESC) and the European Society of Anaesthesiology (ESA). Eur. Heart. J. 35: 2383-2431.

13. Levett D.Z.H., Jack S., Swart M., Carlisle J., Wilson J., Snowden C., Riley M., Danjoux G., Ward S.A., Older P., Grocott M.P.W. 2018. Perioperative Exercise Testing and Training Society (POETTS) Perioperative Cardiopulmonary Exercise Testing (CPET): Consensus Clinical Guidelines on Indications, Organization, Conduct, and Physiological Interpretation. Br. J. Anaesth. 120 (3): 484-500. PMID: 29452805, DOI: 10.1016/j.bja.2017.10.020. 
14. Older P.O., Levett D.Z.H. 2017. Cardiopulmonary Exercise Testing and Surgery. Ann. Am. Thorac. Soc. 1: S74-S83. DOI: 10.1513/AnnalsATS.201610-780FR.

15. Palange P., Ward S.A. 2007. Recommendations on the use of exercise testing in clinical practice. Eur. Respir. J. 29 (1): 185-209. DOI: 10.1183/09031936.00046906.

16. Reeves T., Bates S., Sharp T., Richardson K., Bali S., Plumb J., Anderson H., Prentis J., Swart M., Levett D.Z.H. 2018. Perioperative Exercise Testing and Training Society (POETTS). Cardiopulmonary Exercise Testing (CPET) in the United Kingdom-a National Survey of the Structure, Conduct, Interpretation and Funding. Perioper Med (Lond). 7: 2-8. PMID: 29423173, DOI: 10.1186/s13741-0170082-3.

17. Smith T.B., Stonell C. 2009. Cardiopulmonary exercise testing as a risk assessment method in non-cardio-pulmonary surgery: a systematic review. Anaesthesia. 64 (8): 883-893.

18. Wasserman K. 1993. Preoperative evaluation of cardiovascular reserve in the elderly. Chest. 104 (3): 663-664. PMID: 8365269, DOI: 10.1378/chest.104.3.663.

19. Wasserman K., Hansen J.E., Sue D.Y., Stringer W.W., Sietsema K.E., Sun X-G., Whipp B.J. 2012. Principles of Exercise Testing and Interpretation: 5th edition. Lippincott Williams \& Wilkins. 899 p. ISBN-13: 978-1-60913-899-8.

20. Weisman I.M., Zeballos R.J. 2001. Clinical Exercise Testing. Clin. Chest. Med. 22 (4): 679-701. PMID: 11787659, DOI: 10.1016/s0272-5231(05)70060-5.

\section{ИНФОРМАЦИЯ ОБ АВТОРАХ}

Зарипова Зульфия Абдулловна, кандидат медицинских наук, доцент кафедры анестезиологии и реаниматологии, руководитель центра аттестации и аккредитации Первого Санкт-Петербургского государственного медицинского университета имени академика И.П. Павлова, г. СанктПетербург, Россия

Ткачук Анастасия Сергеевна, клинический ординатор кафедры анестезиологии и реаниматологии Первого СанктПетербургского государственного медицинского университета имени академика И.П. Павлова, г. Санкт-Петербург, Россия

Тишков Артем Валерьевич, кандидат физико-математических наук, доцент, заведующий кафедрой физики, математики и информатики Первого Санкт-Петербургского государственного медицинского университета имени академика И.П. Павлова, г. СанктПетербург, Россия

Страх Любовь Владимировна, ординатор кафедры онкологии Первого СанктПетербургского государственного медицинского университета имени академика И.П. Павлова, г. Санкт-Петербург, Россия

\section{INFORMATION ABOUT THE AUTHORS}

Zulfiia A. Zaripova, Candidate of Science, Associate professor, Head of the certification and accreditation center Pavlov First Saint Petersburg State Medical University

Anastasiya S. Tkachuk, Clinical resident of the Department of anesthesiology and resuscitation Pavlov state medical University of The Ministry of health of Russia

Artem V. Tishkov, Candidate of Science, Docent, Head of Physics, Mathematics and Informatics Department Pavlov First Saint Petersburg State Medical University

Liubov V. Strakh, Resident at the Department of Oncology Pavlov First Saint Petersburg State Medical University 